\title{
Evolvement of best practice in diagnosis and management of idiopathic pulmonary fibrosis
}

\author{
Venerino Poletti \\ Affiliations: Dept of Diseases of the Thorax, Ospedale GB Morgagni, Forlì, Italy.
}

Correspondence: V. Poletti, Dept of Diseases of the Thorax, Ospedale GB Morgagni, via Forlanini 34, Forli, 47100, Italy. E-mail: venerino.polettidgmail.com

0

@ERSpublications

IPF has a poor prognosis but pirfenidone, a new and effective drug, has been approved for use in Europe http://ow.ly/rFqih

Idiopathic pulmonary fibrosis (IPF) is a rare but inevitably progressive and fatal lung disease, with a prognosis that can be worse than many forms of cancer $[1,2]$. There have been a number of important advances and achievements in this field over the past 2 years, which have increased our understanding of how IPF can be managed and brought new hope for patients who have this devastating disease. Until recently, there were no pharmacological treatments approved for patients with IPF in Europe. A major advance in 2011 was the European approval of pirfenidone for adults with mild-to-moderate IPF. Since its introduction, there is now a growing body of evidence regarding the use of pirfenidone in clinical practice, enabling clinicians to discuss some key issues regarding the recommended management of IPF, including the role of earlier diagnosis of IPF, expected clinical outcomes with pirfenidone in the real-world setting, and the role of the multidisciplinary team in diagnosis.

With this background of increasing knowledge and clinical experience in the management of IPF, this issue of the European Respiratory Review includes two articles highlighting the substantial progress that has been made in the management of IPF. These articles are based on presentations from a symposium held at the 2013 European Respiratory Society Annual Congress entitled "Evolvement of best practice in diagnosis and management of IPF". CotTIN and RICHELDI [3] review the neglected evidence in IPF including the role of earlier diagnosis and an update on an ongoing clinical study investigating correlations between lung sounds on auscultation and high-resolution computed tomography images. KREUTER [4] introduces new data analyses regarding pirfenidone, including a per-protocol analysis of the CAPACITY studies, new data from the RECAP extension study and expert centre experience. These articles provide an excellent opportunity for all clinicians involved in the care of patients with IPF to review key concepts and the evolving role of pirfenidone treatment in the management of this disease.

Received: Dec 062013 | Accepted: Dec 072013

Conflict of interest: Disclosures can be found alongside the online version of this article at err.ersjournals.com

Provenance: Publication of this peer-reviewed article was supported by InterMune International AG, Muttenz, Switzerland (article sponsor, European Respiratory Review issue 131).

Copyright CERS 2014. ERR articles are open access and distributed under the terms of the Creative Commons Attribution Non-Commercial Licence 3.0. 


\section{Acknowledgements}

This article is based on the proceedings of a satellite symposium held at the 2013 European Respiratory Society Annual Congress (Barcelona, Spain), which was sponsored by InterMune. I was assisted in the preparation of the article by IntraMed International (Milan, Italy), funded by InterMune.

\section{References}

1 Poletti V, Ravaglia C, Buccioli M, et al. Idiopathic pulmonary fibrosis: diagnosis and prognostic evaluation. Respiration 2013; 86: 5-12.

2 Chilosi M, Carloni A, Rossi A, et al. Premature lung aging and cellular senescence in the pathogenesis of idiopathic pulmonary fibrosis and COPD/emphysema. Transl Res 2013; 162: 156-173.

3 Cottin V, Richeldi L. Neglected evidence in idiopathic pulmonary fibrosis and the importance of early diagnosis and treatment. Eur Respir Rev 2014; 23: 106-110.

4 Kreuter M. Pirfenidone: an update on clinical trial data and insights from everyday practice. Eur Respir Rev 2014; 23: 111-117. 